\title{
How do I audit and receive feedback on my clinical practice?
}

\author{
Julian Marsden, MD* \\ Ignorance more frequently begets confidence than does \\ knowledge.
}

\author{
—Charles Darwin
}

\begin{abstract}
Audit and feedback (A\&F) is a powerful exercise for selfassessment of individual or group practice against best practice. Learning occurs through a structured process, starting by understanding the area of interest, conducting an audit, and then reflecting and creating solutions to identified gaps in care. Although current evidence is limited, the additional time and effort required are believed to result in greater rewards than traditional educational activities as the learning is more directly relevant to patient care.
\end{abstract}

L'ignorance engendre la confiance plus souvent que le fait la connaissance.

\section{-Charles Darwin}

La vérification-rétroaction (A\&F) est un excellent exercice pour l'auto-évaluation de la pratique individuelle ou de groupe par rapport à la pratique exemplaire. L'apprentissage se produit au cours d'un processus structuré qui commence par la compréhension du champ d'intérêt; ensuite, on mène une vérification, puis on réfléchit et on crée des solutions pour les lacunes décelées dans les soins. Bien que les données actuelles soient limitées, nous pensons que le temps et les efforts additionnels requis entraînent de plus grands bénéfices que les activités de formation traditionnelles puisque l'apprentissage est plus directement axé sur les soins aux patients.

Keywords: audit and feedback, education, knowledge translation

\section{CLINICAL SCENARIO}

You are suddenly awake. As your head clears, you recall the 68-year-old man with chest pain you saw during last evening's shift. After your workup, you discharged him to see his family physician in the next week. Should you have investigated further or even had him seen by the internist on call? You convince yourself that you made the right decision but resolve to follow up with the family doctor.

Now fully awake, your thoughts drift. What would your colleagues have done? What can you do to make sure that you are practicing to the best of your abilities? You recall your colleague saying something about A\&F being much better than simply attending a conference and decide to look into this.

\section{WHY SHOULD I KNOW ABOUT A\&F?}

Quite simply, the nature of emergency medicine is too complex to be addressed by any one form of professional development. We must unravel complex presentations despite time constraints and limited information. The autonomous nature of our work means that, for the most part, we are left to our own devices. Most of us informally obtain feedback when we follow up on a patient or discuss a challenging case with colleagues, but these feedback methods are haphazard at best. Even the structured morbidity and mortality round is limited by the usual focus on systematic issues that benefit an entire group rather than the unique context of a given clinician-patient interaction.

The challenge is that our real potential for improvement lies in those areas where "we do not

From the *Department of Emergency Medicine, University of British Columbia, Vancouver, BC.

Correspondence to: Dr. Julian Marsden, Emergency Department, St. Paul's Hospital, 1081 Burrard Street, Vancouver, BC V6Z 1Y6; marsden@ telus.net.

Submitted December 9, 2010; Revised April 15, 2011; Accepted April 18, 2011.

This article has been peer reviewed.

(c) Canadian Association of Emergency Physicians

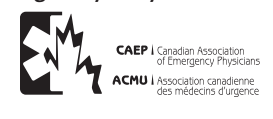


know what it is we do not know." Davis and colleagues found that a better approach includes external feedback such as A\&F. ${ }^{1}$ In a recent survey of 297 emergency physicians, a significant majority indicated that they receive very little feedback on their practice, but almost all $(97.3 \%)$ would welcome it in the belief that this would improve their practice. ${ }^{2}$

\section{WHAT IS A\&F?}

$\mathrm{A} \& \mathrm{~F}$ is "a systematic review of care against explicit criteria and ... where indicated, changes are implemented at an individual, team, or service level and further monitoring is used to confirm improvement." ${ }^{3}$ Although the objective can be for system redesign or for faculty development of the emergency group at large, the focus of this article is at the individual or small-group level. Furthermore, the process can be as extensive as multisource feedback, ${ }^{4}$ which involves a questionnaire-based feedback from several peers, patients, and coworkers, but for ease of application, this article deals with what can be done immediately.

\section{DOES A\&F WORK?}

Reviews indicate that $\mathrm{A} \& \mathrm{~F}$ is a reasonable approach to improve practice. Grimshaw and colleagues reported a median improvement in physician behaviour of $7 \%$ compared to no intervention (range 1.3-16\%). Jamtvedt and colleagues reviewed 118 studies and concluded that $\mathrm{A} \& \mathrm{~F}$ was only mildly to moderately useful compared to no intervention, with a range of improvement from 5 to $16 \%{ }^{6}$ However, one study found a $10 \%$ worsening, whereas another found a $70 \%$, leading to the question of how comparable the studies are. Further confounders include differences in the type and intensity of feedback as well as outcomes measured. Nevertheless, A\&F was beneficial in those settings where tremendous variation in practice approach existed.

\section{HOW DO I UNDERTAKE AN A\&F?}

$\mathrm{A} \& \mathrm{~F}$ can be done by individual physicians, as a smallgroup exercise, or as faculty development for a larger group. A structured approach ${ }^{7,8}$ helps improve the commitment to the work and provides a template for future iterations. Furthermore, the work needs to be one of mutual learning rather than a performance review to facilitate valid feedback and conversations.
This is a critical point because the learning is often experiential and at an emotional level. The following steps are summarized in Table 1:

\section{Table 1. Approach to audit and feedback}

Step 1. Select a topic of interest

Define the area of interest clearly by creating a specific question and/or parameters around the issue.

Examples

1. All chest pain patients over age 60 who were admitted to hospital

2. Did all discharged chest pain patients over age 40 with two negative troponin tests get provocative testing within 2 weeks?

3. All deaths within 24 hours of arrival at hospital

4. How long do patients with chest pain wait in my waiting room? Step 2. Determine best practice and create your audit criteria

Based on a literature review, existing national or regional guidelines, or simply feedback from consultants and colleagues, the next step is to create acceptable measures of care.

Examples

1. Do low-risk chest pain patients who were discharged have a discharge plan?

2. Do chest pain patients at low risk for ACS have repeat ECGs and troponin levels out to 6 hours from the time of ED admission?

3. Do chest pain patients in whom you are ruling out ACS receive at least $80 \mathrm{mg}$ of ASA?

Step 3. Collect data

Determine how you will conduct the search or chart audit and what you will collect. Develop a chart audit form to capture the information. Consider factors that will make your results more

robust but that can be collected efficiently, such as

Patient's age

Chest pain was main presenting complaint

Duration of chest pain

Medication profile

Whether the patient had any ECG abnormalities

Admitted

Referred to internist/cardiologist

For example, contact medical records to pull the charts on the last 10 patients over the age of 40 years who presented with chest pain, were discharged home, and had an ECG performed and a troponin level taken in the ED.

Step 4. Analyze performance against the agreed upon criteria

Answer your question(s) or outline findings for further discussion. What conclusion do you draw from these findings? For example, 5 of the 10 patients did not have a second troponin test done or 4 of the 10 patients had no follow-up arranged.

Step 5. Implement recommendations for improvement

What changes do you plan to make and when? Resolve to adopt an individual action plan or take this to the group to discuss ways to embed appropriate standard care into everyday practice. For example, every chest pain patient over the age of 40 without a clear alternate cause will have 2 troponin tests and 2 ECGs done at least 2 hours apart to rule out an acute event and will have follow-up arranged through their family doctor as soon as possible.

Step 6. Sustain improvements

Repeat the A\&F in 6 months.

$A \& F=$ audit and feedback; $A C S=$ acute coronary syndrome; $A S A=$ acetylsalicylic acid $\mathrm{ECG}=$ electrocardiogram; $\mathrm{ED}=$ emergency department. 


\section{Step 1. Identify a topic or question}

In choosing the topic, it is helpful to know how you wish to approach the A\&F: generate group discussion on a topic such as management of chest pain patients over the age of 50 who get an electrocardiogram (ECG) or address a specific issue such as how many chest pain patients who are discharged home are readmitted or die within 30 days. Both strategies can lead to important findings, but the latter will make it easier to conduct the audit and produce more tangible results. It is recommended where there is good evidence about clinical practice.

\section{Step 2. Determine best practice and create your audit criteria}

Based on a literature review, existing national or regional guidelines (e.g., Guidelines International Network: $<$ http://www.g-i-n.net/library/international-guidelineslibrary>; the American National Guideline Clearinghouse: < http://www.guidelines.gov/ $>$; or the Canadian CMA Infobase: <http://www.cma.ca/index.php/ci_id/ 54316/la_id/1.htm $>$ ), or simply feedback from consultants and colleagues, the next step is to create acceptable measures of care, such as giving acetylsalicylic acid to all patients with chest pain who get an ECG. These audit criteria help clarify the overall purpose of the audit and explicitly define what is being measured. Creating specific parameters around the issue, such as patient age group, presenting complaint, and admitted versus discharged patients, will also help with the audit.

\section{Step 3. Collect data}

$\mathrm{A} \& \mathrm{~F}$ is driven by data, but its collection can be burdensome, even with clear audit criteria. Decisions on what data will be collected, where to look for data, and who will do the data collection must be made. Potential sources of bias must be considered: who you will include or exclude in the audit? For example, using criteria cutoffs such as age, socioeconomic status, and number of comorbid conditions may all lead to different findings. Starting with younger, healthier patients and moving to the more complicated cases may be best as each group may have different outcomes that would be lost if lumped together. Ethical issues must also be considered, along with staff and patient confidentiality. It may be necessary to discuss any sensitive topics with the local Research Ethics Committee.

\section{Step 4. Analyze performance against the agreed upon criteria}

Comparing the results of the data collection with the audit criteria will allow you to identify and characterize the gaps in care and, ideally, generate discussion around possible solutions. Each gap needs to be well defined, followed by a discussion of the significance of the findings and the feasibility of necessary improvements. For example, correcting a minor delay in treatment might require too many resources to achieve. Sharing the results with all appropriate parties early in the process will involve them in the discussion and help implement any resultant recommendations.

\section{Step 5. Implement recommendations for improvement}

Implementing the suggested recommendations is a challenging but essential part of the $\mathrm{A} \& \mathrm{~F}$ cycle. Using an action plan to record the recommendations is good practice and should include assigning timelines and most responsible people. It may only require one champion of change to initiate change, but most recommendations also require the engagement and effort of multiple stakeholders to be successful. A useful and easy to read resource is John Kotter's book, Our Iceberg Is Melting: Changing and Succeeding Under Any Conditions. ${ }^{9}$

\section{Step 6. Sustain improvements}

The A\&F should be repeated to demonstrate that the changes have been implemented and that they led to improved patient care and individual or group satisfaction.

\section{WHAT FACTORS WILL INCREASE THE VALUE OF AN A\&F?}

A number of factors increase the success of A\&F.10,11 Basing the feedback on chart data is important; equally important is considering how the feedback is provided and the consequences of that feedback. The emotional involvement is higher and the ability to recall details is greater when the $A \& F$ is performed soon after the event(s). The effect of the feedback is greater if provided by a respected member of the group, such as the head of the department, although a local 
champion can work. Having ongoing feedback as part of the group's continuing professional development demonstrates that $\mathrm{A} \& \mathrm{~F}$ is an important part of the practice improvement culture.

Other factors that have been found to increase the success of changing practice patterns are creating a culture where the focus is on improving patient care rather than focusing on (shaming) clinicians; giving more frequent feedback; providing written feedback rather than verbal; and, when possible, providing specific recommendations.

\section{CONCLUSION}

$\mathrm{A} \& \mathrm{~F}$ is one form of continuing professional development that has the potential to make significant improvements in patient care and provider satisfaction. It can be done at an individual, small-group, or largegroup level. Having a structured approach with a careful consideration of how to carry out the $A \& F$ will help optimize its value. Although it requires more effort than other forms of continuing professional development, its proactive approach can empower individuals and groups to maximize their learning potential and to improve emergency patient care.

Competing interests: None declared.

\section{REFERENCES}

1. Davis DA, Mazmanian PE, Fordis M, et al. Accuracy of physician self-assessment compared with observed measures of competence. A systematic review. 7AMA 2006;296:1094102, doi:10.1001/jama.296.9.1094.

2. Lavoie CF, Plint AC, Clifford TJ, et al. "I never hear what happens, even if they die" : a survey of emergency physicians about outcome feedback. CFEM 2009;11:523-8.

3. Foy R, Eccles MP, Jamtvedt G, et al. What do we know about how to do audit and feedback? Pitfalls in applying evidence from a systematic review. BMC Health Serv Res 2005;5:50. doi:10.1186/1472-6963-5-50.

4. Sargeant J, Mann K, Sinclair D, et al. Challenges in multisource feedback: intended and unintended outcomes. Med Educ 2007;41:583-91, doi:10.1111/j.1365-2923. 2007.02769.x.

5. Grimshaw JM, Shirran L, Thomas R, et al. Changing provider behaviour: an overview of systematic reviews of interventions. Med Care 2001;39 Suppl 2:II2-45.

6. Jamtvedt G, Young JM, Kristoffersen DT, et al. Audit and feedback: effects on professional practice and health care outcomes. Cochrane Database Syst Rev 2006;(2)CD000259, doi:10.1002/14651858.CD000259.pub2.

7. National Institute of Clinical Excellence. Principles for best practice in clinical audit. London: National Institute of Clinical Excellence; 2002. Available at: http://www.nelh. nhs.uk/nice_bpca.asp (accessed November 15, 2010).

8. Godwin M. Conducting a clinical practice audit. Fourteen steps to better patient care. Can Fam Phys 2001;47:2331-3.

9. Kotter J. Our iceberg is melting: changing and succeeding under any conditions. NewYork: St. Martin's Press; 2005.

10. Hysong SJ. Meta-analysis: audit and feedback features impact effectiveness on care quality. Med Care 2009;47: 356-63, doi:10.1097/MLR.0b013e3181893f6b.

11. Hysong SJ, Best RG, Pugh JA. Audit and feedback and clinical practice guideline adherence: making feedback actionable. Implement Sci 2006;1:9, doi:10.1186/1748-5908$1-9$. 